\section{() OPEN ACCESS}

\title{
Natriuretic peptide-guided treatment for heart failure: a systematic review and meta-analysis
} Julie McLellan $\odot$, Clare R Bankhead, Jason L Oke,
F D Richard Hobbs, Clare J Taylor, Rafael Perera ๑

10.1136/bmjebm-2019-111208

- Additional material is published online only. To view please visit the journal online (http://dx.doi.org/ 10.1136/bmjebm-2019111208).

Nuffield Department of Primary Care Health Sciences, University of Oxford, Oxford, UK

Correspondence to: Dr Clare R Bankhead, Nuffield Department of Primary Care Health Sciences, University of Oxford, Oxford OX2 6GG, UK; clare.bankhead@phc. ox.ac.uk

\section{SLinked}

- http://dx.doi.org/10.1136/ bmjebm-2019-111183

- http://dx.doi.org/10.1136/ bmjebm-2019-111243

\section{Check for updates}

(c) Author(s) (or their employer(s)) 2020. Re-use permitted under CC BY-NC. No commercial re-use. See rights and permissions. Published by BMJ.

To cite: McLellan J, Bankhead CR, Oke JL, et al. BMJ Evidence-Based Medicine 2020;25:33-37.

\section{Abstract}

Background GUIDE-IT, the largest trial to date, published in August 2017, evaluating the effectiveness of natriuretic peptide (NP)-guided treatment of heart failure (HF), was stopped early for futility on a composite outcome. However, the reported effect sizes on individual outcomes of all-cause mortality and HF admissions are potentially clinically relevant. Objective This systematic review and metaanalysis aims to combine all available trial level evidence to determine if NP-guided treatment of $\mathrm{HF}$ reduces all-cause mortality and $\mathrm{HF}$ admissions in patients with $\mathrm{HF}$.

Study selection Eight databases, no language restrictions, up to November 2017 were searched for all randomised controlled trials comparing NPguided treatment versus clinical assessment alone in adult patients with HF. No language restrictions were applied. Publications were independently double screened and extracted. Fixed-effect metaanalyses were conducted.

Findings 89 papers were included, reporting 19 trials (4554 participants), average ages 62-80 years. Pooled risk ratio estimates for all-cause mortality (16 trials, 4063 participants) were 0.87 , 95\% CI 0.77 to 0.99 and $0.80,95 \%$ CI 0.72 to 0.89 for HF admissions (11 trials, 2822 participants). Sensitivity analyses, restricted to low risk of bias, produced similar estimates, but were no longer statistically significant.

Conclusions Considering all the evidence to date, the pooled effects suggest that NP-guided treatment is beneficial in reducing HF admissions and all-cause mortality. However, there is still insufficient high-quality evidence to make definitive recommendations on the use of NPguided treatment in clinical practice.

Trial registration number Systematic Review Cochrane Database Number: CD008966.

\section{Introduction}

Natriuretic peptides (NP) are released by the myocardium in response to pressure or fluid overload and are raised in patients with heart failure (HF). NP testing is currently used in diagnosis, but its role in guiding $\mathrm{HF}$ treatment remains controversial. In August 2017, the long-awaited GUIDE-IT study was published by Felker and colleagues. ${ }^{1}$ It is the largest study of NP monitoring to date, recruiting 894 of the planned 1100 participants, and was stopped early due to futility.
Lack of efficacy for the biomarker-guided treatment group compared with usual care was based on the primary end point, a composite outcome of $\mathrm{HF}$ admission and cardiovascular mortality. The conclusion that NP-guided treatment is not more effective than usual care has been rapidly disseminated with the paper viewed over 12000 times in the first 4 months following publication and 22000 to date. While the authors did not comment on the implications for practice, the emerging consensus favours an interpretation that "any plausible effects of an intervention are unlikely to be worthwhile.' ${ }^{2}$ In response to the publication of the GUIDE-IT study, ${ }^{1}$ Bajaj et $a l^{3}$ published a research letter with a meta-analysis reporting all-cause mortality as significantly lower in the NP-guided group. This rapid analysis only collated evidence from six studies, obtained from one database for a single outcome. Although there tends to be good consistency between the single largest study and pooled estimates from a full systematic review, ${ }^{4}$ it is good practice to frame the newest evidence in context of the previous information. ${ }^{5}$ This helps validate new findings and if done by updating a previous meta-analysis, will allow for higher precision of the estimates, reduced variability and increased generalisability.

Systematic reviews carried out in this area have proved inconclusive, providing conflicting results. Five out of 11 systematic reviews with metaanalyses have demonstrated that NP-guided treatment reduces all-cause mortality in patients with HF compared with usual clinical care, ${ }^{6-10}$ while the more recent systematic reviews, including two with individual patient data, have published metaanalyses contradicting this finding and showing no effect. ${ }^{11-14}$ One of the individual patient data analyses, Troughton et $a l,{ }^{11}$ was able to adjust for patient characteristics and compared time to allcause mortality between NP-guided and clinicallyguided treatment groups and then reported a reduction in all-cause mortality. The most comprehensive meta-analysis ${ }^{12}$ to date, prior to the GUIDE-IT study, ${ }^{1}$ included 3660 participants from 18 studies. Data from GUIDE-IT ${ }^{1}$ increases the amount of evidence for all-cause mortality by over 25\% (previously 3169 participants from 15 studies) and for HF hospital admission by 45\% (previously 1928 participants from 10 studies). These two outcomes, along with adverse events, are the only outcomes where new evidence has been obtained in comparison to the most comprehensive metaanalysis to date. Therefore, this study aims to incorporate the GUIDE-IT study into an update 
of this meta-analysis ${ }^{12}$ to determine if the question of whether NP-guided treatment of patients with HF improves all-cause mortality, HF hospital admissions and adverse events, compared with clinical assessment alone, has been finally answered.

\section{Methods}

This systematic review and meta-analysis was conducted in accordance with the Preferred Reporting Items for Systematic reviews and Meta-analyses (PRISMA) guidelines. ${ }^{15}$ The protocol was published in the Cochrane library. ${ }^{16}$

\section{Data searches and study selection}

The same search strategy was used as in the original review ${ }^{13}$ (online supplementary appendix 1). The following databases were searched up to 27 November 2017: Cochrane Central Register of Controlled Trials, MEDLINE, EMBASE, Database of Abstracts of Reviews of Effects, NHS Economic Evaluation Database, Science Citation Index Expanded and the Conference Proceedings Citation Index on Web of Science, Clinical trials Registry and the WHO International Clinical Trials registry Platform. No restriction was made in terms of year published, language or publication status. All publications were independently double screened first by title and abstract and then by full text (JM, CB, AF). Discrepancies were resolved by consensus.

All randomised controlled trials (RCT) of NP-guided treatment of HF, both in and out of hospital setting, reporting a clinical outcome were included. NP is a collective term for N-terminal pro B-type natriuretic peptide (NT-proBNP) and B-type natriuretic peptide (BNP). Studies compared NP-guided treatment to treatment guided by clinical assessment alone. There was no restriction on the length of follow-up. Participants less than 18 years old were excluded. The outcomes for the systematic review were prespecified. The primary outcome was all-cause mortality, while HF admission was the secondary outcome.

\section{Data extraction and quality assessment}

For each included RCT, data were extracted independently by two people and any discrepancies resolved by consensus (JM, CB). Data extracted included baseline characteristics (gender, age, New York Heart Association (NYHA) classification and left ventricular ejection fraction (LVEF), setting, inclusion and exclusion criteria and descriptions of the intervention and clinical assessment arms including: NP biomarker used, target NP level and drug algorithms for uptitration of HF medication. Assessment of the methodological quality of the studies was made using the Cochrane Risk of bias tool. ${ }^{17}$ The outcomes were extracted as absolute number of events. If a study compared more than one type of control group, then the intervention group data were split equally between the control groups for both outcome events and sample size. Reporting bias was assessed using funnel plots.

\section{Data synthesis and analysis}

Trial level data were pooled using Review Manager V.5.3. ${ }^{18}$ For binary outcomes, data were combined using a Mantzel-Haenzel fixed-effect model to obtain a summary estimate of the risk ratio (RR) with 95\% CI. The I ${ }^{2}$ statistic was used to assess the level of statistical heterogeneity. ${ }^{17}$ Where substantial heterogeneity $\left(\mathrm{I}^{2} \geq 50 \%\right)$ was present, a random-effects model was used to test the robustness of the findings.

Subgroup analyses were considered for age, NYHA class, baseline NP, target NP, achieved NP decrease, setting, gender, LVEF, duration of FU, control type and biomarker used. Blinding of outcome assessment and attrition were judged to potentially

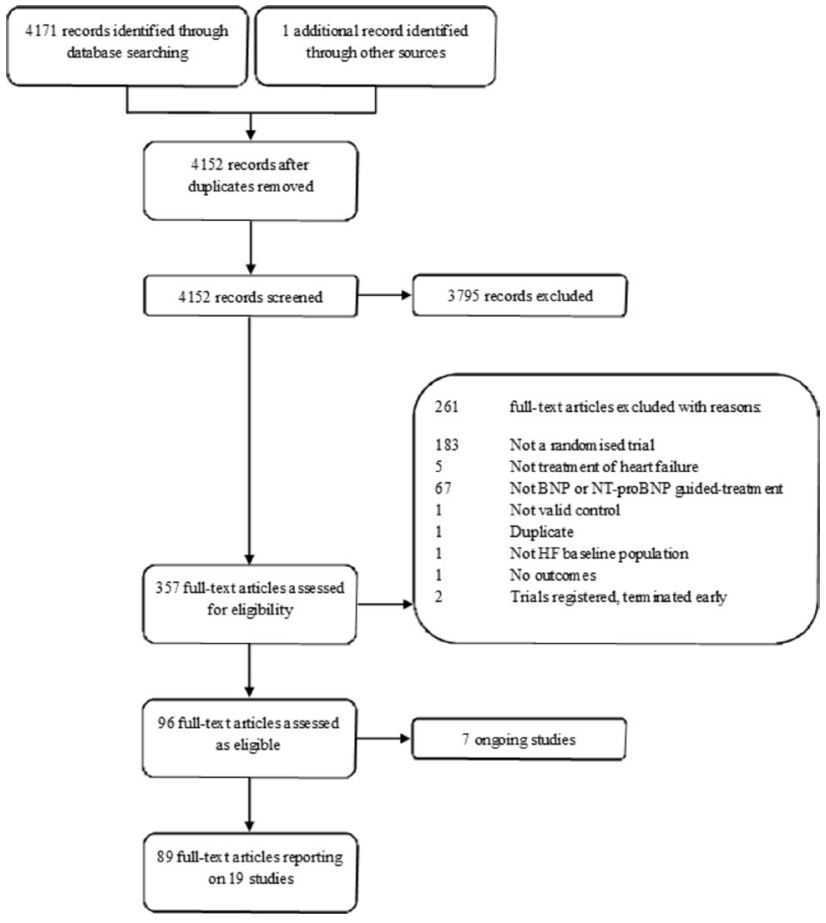

Figure 1 Flowchart of study selection. BNP, B-type natriuretic peptide; $\mathrm{HF}$, heart failure; NT-proBNP, N-terminal pro B-type natriuretic peptide.

impact on the pooled results and therefore sensitivity analyses were conducted restricted to studies with low risk of bias in these two domains.

\section{Results}

From 4152 references (updated and original search), 357 full texts were assessed, and 19 studies involving a total of 4554 participants met the inclusion criteria (figure 1). This update to the Cochrane systematic review ${ }^{13}$ identified one new study: the GUIDE-IT study. ${ }^{1}$ The recently published PRIMA II study ${ }^{19}$ was excluded as this aimed to reduce NP level between hospital admission and discharge in patients with acute HF rather than ongoing NP-guided treatment. Baseline characteristics of included RCTS and patients are given in online supplementary appendices 2 and 3. Each of the 19 studies reported on one or more of the included outcomes. Two of the 19 studies had three comparison arms comparing NP-guided treatment both to clinical assessment and to usual care.

\section{Outcomes}

Sixteen studies $(n=4063)$ reported results that could be pooled for all-cause mortality. During follow-up, 331 out of 1929 participants (18\%) died in the NP-guided treatment groups compared with 445 out of 2134 (22\%) in the control groups. When pooled, the evidence favours the NP-guided treatment (RR 0.87, 95\% CI 0.77 to 0.99 ). Heterogeneity was low indicating consistency across the included studies $\left(\mathrm{I}^{2}=11 \%\right)$ (figure 2).

Eleven studies with 2822 participants reported on HF admission. Out of 1304 participants, 366 (28\%) experienced a HF admission in the NP-guided treatment groups compared with 544 out of 1518 (36\%) participants in the control groups. Overall, the pooled evidence showed an effect favouring NP-guided treatment (RR $0.80,95 \%$ CI 0.72 to 0.89 ) (figure 3 ). Heterogeneity was substantial $\left(\mathrm{I}^{2}=67 \%\right)$. The robustness of this finding was tested by converting 


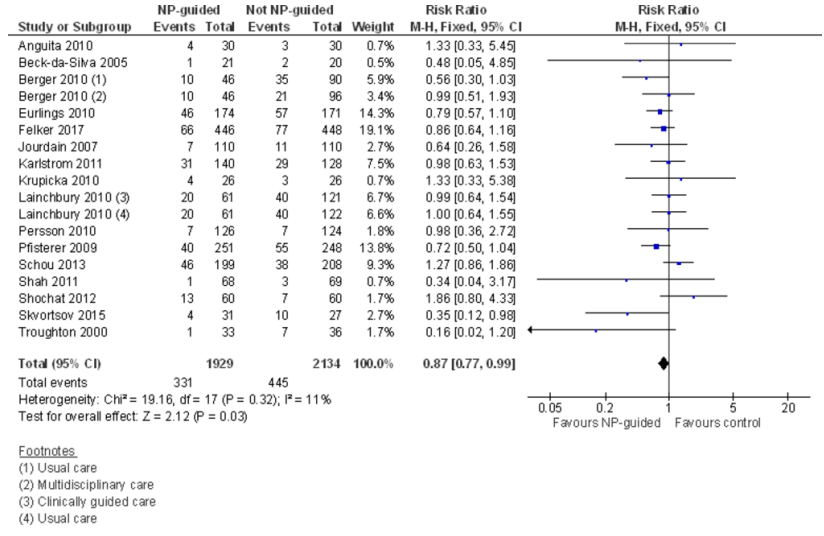

Figure 2 Forest plot comparing NP-guided treatment versus control for all-cause mortality. NP, natriuretic peptide.

to a random-effects model; the effect remained consistent (RR $0.70,95 \%$ CI 0.56 to 0.88 ).

Adverse events were reported as low and similar between groups in the GUIDE-IT study; the data were added to the tabulated data for all studies (online supplementary appendix 4). Data were not pooled for this outcome as it was possible to have multiple events per individual.

Quality assessment of the studies is summarised in online supplementary appendices 5 and 6 . The risk of bias was assessed to be high or unclear for the majority of studies across all domains, except selection bias. Blinding of outcome assessment was judged to be poor with only $36 \%$ of studies assessed as low risk of bias. Attrition bias was assessed as low risk in $42 \%$ of studies.

Except for age, duration of follow-up and biomarker used, it was not possible to explore subgroups within the study populations. Data were reported as totals for whole study or in varying categories or averages for intervention and control groups.

Although the GUIDE-IT study ${ }^{1}$ reported subgroup data for participants above and below 75, it was only for the study's primary composite outcome. Therefore, the analysis in the original systematic review ${ }^{13}$ remained the same, which found uncertainty in the evidence for NP-guided treatment for all-cause mortality when examining under or over 75 years of age and a reduction in HF admission.

Subgroup analyses were completed for duration of the intervention and NP biomarker used. For duration of the intervention, this shows that both at $\leq 1$ and 1-2years, there was a potential reduction for all-cause mortality, but the evidence showed uncertainty at $>2$ years. The effect of duration on HF admission showed

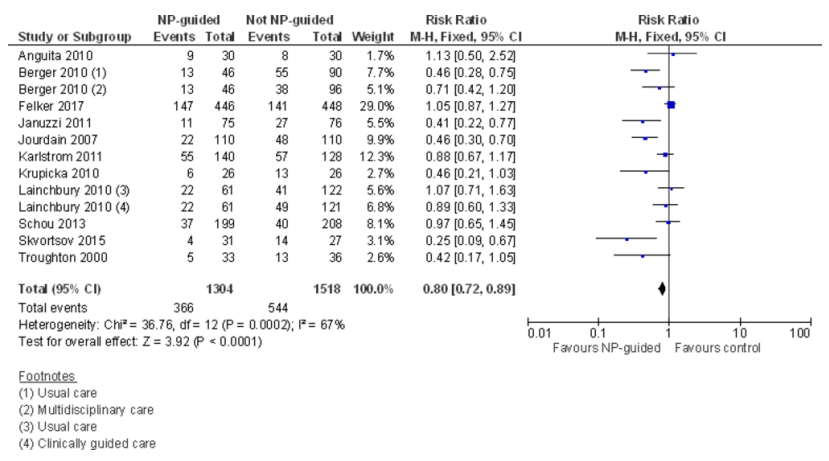

Figure 3 Forest plot comparing NP-guided treatment versus control for heart failure admission. NP, natriuretic peptide. a similar trend for each subgroup for the same durations (online supplementary appendices 7 and 8).

Analyses completed for the biomarker used suggested no difference between the biomarker used and between the main findings for both all-cause mortality and HF admissions. However, the subgroups for both biomarkers for all-cause mortality were no longer statistically significant (online supplementary appendices 9 and 10).

Sensitivity analyses were completed restricting studies to those with low risk of bias for blinding of outcome assessment and attrition. The GUIDE-IT study ${ }^{1}$ was assessed to be low risk of bias for blinding of the outcome assessment and therefore adding these data in the sensitivity analyses found for both all-cause mortality and HF admission the effect continued to favour NP-guided treatment, but was no longer statistically significant (all-cause mortality: RR 0.92, CI 0.79 to 1.06 ; HF admission: RR 0.92, CI 0.81 to 1.4$)$.

For the risk of attrition bias, the GUIDE-IT study ${ }^{1}$ was assessed to be unclear and therefore provided no additional data to the original systematic review. ${ }^{13}$ Analyse restricted to studies with low risk of attrition bias showed similar results to blinding of outcome assessment for all-cause mortality and HF admissions, though it was no longer statistically significant for all-cause mortality.

\section{Discussion}

This meta-analysis, including all the available evidence, indicates that NP-guided treatment can improve all-cause mortality by $13 \%$ and reduce $\mathrm{HF}$ admissions by $20 \%$. As expected, the addition of the GUIDE-IT study ${ }^{1}$ data, which increased the total number of participants included by $24 \%$, substantially increased the precision of the estimates. It corroborates the conclusion of all previous meta-analyses, bar Pufulete et $a l,{ }^{14}$ including the 2016 Cochrane systematic review ${ }^{13}$ for HF admissions which showed the intervention to be beneficial (online supplementary appendix 11). Perhaps unexpectedly, given that the study stopped early for futility, this meta-analysis contradicts recent previous reviews including the 2016 Cochrane systematic review ${ }^{13}$ and finds that the intervention is also beneficial for all-cause mortality (online supplementary appendix 11). Although the point estimate for the effect is the same as the 2016 Cochrace systematic review, ${ }^{13}$ with or without the GUIDE-IT study, the increase in precision means that the 95\% $\mathrm{CI}$ is consistent with a significant reduction in all-cause mortality (change from CI (0.76 to 1.01) to CI (0.77 to 0.99)) on pooled data. A reduction in all-cause mortality of 13\%, as shown in this metaanalysis, is a clinically important finding.

Clinical guidelines for $\mathrm{HF}$ management ${ }^{20-22}$ vary in their recommendations on NP-guided management for patients with HF reflecting the conflicting evidence in this area of research. The GUIDE-IT study ${ }^{1}$ had aimed to remove this uncertainty by recruiting an appropriate number of participants to detect a difference in clinically meaningful endpoints. Of relevance, the point estimate for all-cause mortality reported by the GUIDE-IT study ${ }^{1}$ showed the intervention to be marginally more effective (RR 0.86, CI 0.62 to 1.20 ) compared with that found in this meta-analysis. A sample size calculation indicates that in order to show an effect size of 13\% reduction in all-cause mortality from a baseline risk of approximately 15\% (as in the GUIDE-IT study ${ }^{1}$ ), a single study would need to include in excess of 10000 participants to be sufficiently powered $(\geq 80 \%)$. This meta-analysis currently includes just over 4000 participants. This is likely to have made all-cause mortality, as a single outcome, prohibitively expensive for the GUIDE-IT study justifying their use of a composite outcome. New 
studies are needed for even pooled evidence to have sufficient power.

The GUIDE-IT authors suggest that the lack of effect may be due to a similar reduction in NP levels in both the intervention and control arms partly mediated by the control group having a higher number of clinic visits than usual as participants were managed in centres with HF expertise. The authors describe the study population as 'high-risk' and eligibility criteria included a $\mathrm{HF}$ event in the last 12 months (hospitalisation, $\mathrm{A}+\mathrm{E}$ attendance or intravenous diuretic) and an NT-proBNP level over $2000 \mathrm{pg} / \mathrm{mL}$ in the prior 30 days. However, many of the other studies included in the meta-analysis recruited participants with NYHA class IIIIV, recent hospital admission or a markedly raised NP level. It is therefore reasonable to consider the studies together.

Nevertheless, the evidence for this effect is not yet robust, as indicated by the subgroup analysis comparing the type of NP biomarker and the sensitivity analyses restricting the evidence to studies assessed as having low risk of outcome assessment bias and low risk of attrition bias. There is still not sufficient evidence to conclude definitively on the effectiveness of NP-guided treatment in the management of $\mathrm{HF}$, and particularly in less severely affected patients, such as those managed in primary care. Reporting of patient and study characteristics needs to be broader and more detailed to allow further exploration of study populations. Many of the interventions evaluated in the trials are multifaceted and as yet, we do not have clear evidence on the mechanism of action behind NP-guided treatment and which factors, if any, drive the change in salient health outcomes in NP-guided management of HF. Improved HF outcomes could be a result of clinical support indirectly intensified by NP-guided treatment rather than simply the monitoring of NP levels. ${ }^{23}$ Identification of key components within interventions linked to improving HF outcomes is needed. Research by Oke et $a l^{24}$ attempted to provide information about the critical features of NP-guided monitoring of HF to reduce HF hospitalisation and suggested that interventions could be simplified. While unable to unequivocally determine an optimal intervention, they suggest that future intervention synthesis incorporating more data may provide clear procedural details of the essential elements of NP-guided monitoring.

\section{Limitations of the study}

While a thorough search with no date or language restrictions was performed, it is possible some studies may have been overlooked in searching and study selection. It was not possible to include data from one study for the primary outcome. Subgroups analyses of the data were limited due to the level of variation in how data were reported. In particular, it was not possible to complete subgroup analysis for LVEF which would have provided an indication of whether patients had HF with preserved ejection fraction (HFpEF) or HF with reduced ejection fraction (HFrEF). Studies did not define themselves as HFpEF or HFrEF, but all studies, at baseline, had either had an average $\mathrm{LVEF}<46 \%$ or inclusion criteria for the study of $<40 \%$ (bar Maeder et $a l,{ }^{25}$ though this study was not included in the meta-analyses). Subgroup analysis by age was possible, but only for three studies who reported data by age. However, since average ages were reported for each study, this analysis should be viewed with caution as it may mask different population distributions found across the included studies. To have an adequate exploration of the impact that age has on these outcomes, individual patient data arising from these studies would be required. Analysis of publication bias was limited due to the lack of studies. However, there is a suggestion of bias with a lack of smaller studies showing an effect favouring the control for the outcome of all-cause mortality (online supplementary appendix 12).

\section{Conclusion}

The current pooled evidence indicates a beneficial effect of NP-guided therapy on all-cause mortality and on HF admissions. However, despite the publication of the GUIDE-IT study, ${ }^{1}$ the largest in this field, there is still insufficient evidence to reach a definitive conclusion on the effectiveness of NP-guided treatment in the management of HF.

Acknowledgements We would like to thank Nia Roberts (Information specialist, Bodleian Library, University of Oxford) for completing the library search and Alice Fuller (Research Officer, University of Oxford) for assisting with the title/abstract screening.

Contributors JM, RP and CB conceived and designed the study. $\mathrm{JM}$ and $\mathrm{CB}$ did the data screening, data extraction and quality assessment. JM, RP and CB did the statistical analyses. FDRH and CJT provided clinical expertise and input. All authors interpreted the data and drafted, critically appraised and approved the final manuscript.

Funding This work was supported by funds for independent research funded by the National Institute for Health Research (NIHR) under the Programme Grants for Applied Research programme (RP-PG-1210-12003). RP and CB are supported by the NIHR Biomedical Research Centre, Oxford. FDRH acknowledges part-funding from the NIHR School for Primary Care Research, NIHR CLARHC Oxford, NIHR Oxford BRC and NIHR Oxford DEC. CJT is supported by an NIHR Academic Clinical Lectureship at the University of Oxford.

Disclaimer The views expressed are those of the authors and not necessarily those of the NIHR or the Department of Health). The study funders did not have any role in the study design, analysis or interpretation of data, in writing of the report or in the decision to submit the article for publication. All researchers were independent from funders.

Competing interests All authors have had financial support from the National Institute for Health Research (NIHR) for the submitted work. RH declared research support for a trial of HF diagnosis from Roche Diagnostics by supply of BNP assays in 2010/11. RP is an Associate Editor for BMJ Evidence-Based Medicine.

\section{Patient consent for publication Not required.}

Provenance and peer review Not commissioned; externally peer reviewed.

Open access This is an open access article distributed in accordance with the Creative Commons Attribution Non Commercial (CC BY-NC 4.0) license, which permits others to distribute, remix, adapt, build upon this work noncommercially, and license their derivative works on different terms, provided the original work is properly cited, appropriate credit is given, any changes made indicated, and the use is non-commercial. See: http://creativecommons.org/licenses/by$\mathrm{nc} / 4.0 /$.

\section{ORCID iDs}

Julie McLellan http://orcid.org/0000-0002-2868-8631

Rafael Perera http://orcid.org/0000-0003-2418-2091 


\section{References}

1. Felker GM, Anstrom KJ, Adams KF, et al. Effect of Natriuretic PeptideGuided Therapy on Hospitalization or Cardiovascular Mortality in High-Risk Patients With Heart Failure and Reduced Ejection Fraction: A Randomized Clinical Trial. JAMA 2017;318:713-20.

2. Glasziou $P$, Chalmers I. How systematic reviews can reduce waste in research. 29 ocotber 2015 ed. BMJ Opinion 2015.

3. Bajaj NS, Patel N, Prabhu SD, et al. Effect of NT-proBNP-Guided Therapy on All-Cause Mortality in Chronic Heart Failure With Reduced Ejection Fraction. J Am Coll Cardiol 2018;71:951-2.

4. Glasziou PP, Shepperd S, Brassey J. Can we rely on the best trial? A comparison of individual trials and systematic reviews. BMC Med Res Methodol 2010;10:23.

5. Young C, Horton R. Putting clinical trials into context. Lancet 2005;366:107-8.

6. Felker GM, Hasselblad V, Hernandez AF, et al. Biomarker-guided therapy in chronic heart failure: a meta-analysis of randomized controlled trials. Am Heart J 2009;158:422-30.

7. Li P, Luo Y, Chen YM. B-type natriuretic peptide-guided chronic heart failure therapy: a meta-analysis of 11 randomised controlled trials. Heart Lung Circ 2013;22:852-60.

8. Li Y, Pei H, Zhou X, et al. Efficacy, modifiable factors to efficacy, safety of B-type natriuretic peptide-guided heart failure therapy: A meta-analysis. Cardiology 2014:66.

9. Porapakkham P, Porapakkham P, Zimmet H, et al. B-type natriuretic peptide-guided heart failure therapy: A meta-analysis. Arch Intern Med 2010;170:507-14.

10. Savarese G, Trimarco B, Dellegrottaglie S, et al. Natriuretic peptide-guided therapy in chronic heart failure: a meta-analysis of 2,686 patients in 12 randomized trials. PLoS One 2013;8:e58287.

11. Troughton RW, Frampton CM, Brunner-La Rocca HP, et al. Effect of Btype natriuretic peptide-guided treatment of chronic heart failure on total mortality and hospitalization: an individual patient meta-analysis. Eur Heart J 2014;35:1559-67.

12. Xin W, Lin Z, Mi S. Does B-type natriuretic peptide-guided therapy improve outcomes in patients with chronic heart failure? A systematic review and meta-analysis of randomized controlled trials. Heart Fail Rev 2015;20:69-80.

13. McLellan J, Heneghan CJ, Perera R, et al. B-type natriuretic peptideguided treatment for heart failure. Cochrane Database Syst Rev 2016;12:Cd008966
14. Pufulete M, Maishman R, Dabner L, et al. Effectiveness and cost-effectiveness of serum B-type natriuretic peptide testing and monitoring in patients with heart failure in primary and secondary care: an evidence synthesis, cohort study and cost-effectiveness model. Health Technol Assess 2017;21:1-150.

15. Moher D, Liberati A, Tetzlaff J, et al. PRISMA Group. Preferred reporting items for systematic reviews and meta-analyses: the PRISMA statement. BMJ 2009;339:b2535.

16. Kearley KE, Lucy Wright F, Tyndel S, et al. B-type natriuretic peptideguided treatment for heart failure. $2011 \mathrm{http}$ ://onlinelibrary.wiley.com/doi/ 10.1002/14651858.CD008966/full (accessed 21 Dec 2017).

17. Higgins JPT, Green S, eds. Cochrane Handbook for Systematic Reviews of Interventions, Version 5.1.0 [updated March 2011]: Cochrane Collaboration.

18. The Nordic Cochrane Centre [program]. Review Manager (RevMan) Version 5.3 Copenhagen, 2014.

19. Stienen S, Salah K, Moons AH, et al. Rationale and design of PRIMA II: A multicenter, randomized clinical trial to study the impact of in-hospital guidance for acute decompensated heart failure treatment by a predefined NT-PRoBNP target on the reduction of readmIssion and Mortality rAtes. Am Heart J 2014;168:30-6.

20. Excellence NIfHaC. Chronic heart failure: the management of chronic heart failure in adults in primary and secondary care (CG108. London: National Clinical Guideline Centre, 2010.

21. Ponikowski P, Voors AA, Anker SD, et al. 2016 ESC Guidelines for the diagnosis and treatment of acute and chronic heart failure: The Task Force for the diagnosis and treatment of acute and chronic heart failure of the European Society of Cardiology (ESC)Developed with the special contribution of the Heart Failure Association (HFA) of the ESC. Eur Heart J 2016;37:2129-200.

22. Ezekowitz JA, O’Meara E, McDonald MA, et al. 2017 Comprehensive Update of the Canadian Cardiovascular Society Guidelines for the Management of Heart Failure. Can J Cardiol 2017;33:1342-433.

23. Chioncel 0, Collins SP, Greene SJ, et al. Natriuretic peptide-guided management in heart failure. $J$ Cardiovasc Med 2016;17:556-68.

24. Oke J, Clements A, McLellan J, et al. Essential components in natriuretic peptide-guided management of heart failure: an intervention synthesis. Open Heart 2018;5:e000826.

25. Maeder MT, Rickenbacher P, Rickli H, et al. N-terminal pro brain natriuretic peptide-guided management in patients with heart failure and preserved ejection fraction: findings from the Trial of Intensified versus standard medical therapy in elderly patients with congestive heart failure (TIME-CHF). Eur J Heart Fail 2013;15:1148-56. 